\title{
Assessment of an Ultrasound-based Scoring System for Triaging Ovarian Tumors in Symptomatic Women
}

\author{
Rosendo Galván, Juan Luis Alcázar, Pedro Royo, Matías Jurado, José Ángel Mínguez, Manuel García \\ Manero, Carmen Laparte, Guillermo López García \\ Department of Obstetrics and Gynecology, Clinica Universitaria de Navarra, School of Medicine, University of Navarra, Pamplona \\ Spain
}

Correspondence: Juan Luis Alcázar, MD, Department of Obstetrics and Gynecology, Clinica Universitaria de Navarra Avenida Pio XII, 3631008 Pamplona, Spain, Phone: 34-948-255400, Fax: 34-948-296500, e-mail: jlalcazar@unav.es

\begin{abstract}
Objective: To evaluate prospectively an ultrasound-based scoring system as a method for triaging symptomatic women presenting with an adnexal mass for surgical treatment.

Methods: 151 symptomatic women scheduled for surgical treatment at our institution were included in this prospective study. Patients were evaluated by transvaginal power Doppler ultrasound prior to surgery. Patients were classified as low-risk or high-risk for malignancy according an ultrasound-based scoring system. Patients with low risk for malignancy were scheduled for laparoscopy and patients for high risk for malignancy were scheduled for laparotomy. Some patients with high-risk were scheduled for advanced oncologic laparoscopic surgery. Patients with low risk but tumor size $\geq 10 \mathrm{~cm}$ were scheduled for laparotomy.

Results: 82 women presented with pelvic pain, 8 had uterine bleeding and 61 referred symptoms suggestive for ovarian malignancy, such as abdominal swelling, bloating and abdominal discomfort. 75 (49.7\%) masses were considered as "low-risk" and treated by laparoscopy in 58 cases and by laparotomy in 7 cases, because emergency or associated pathology (All tumors were benign). 76 (50.3\%) masses were considered as "high-risk" and all treated by laparotomy (56 malignant and 4 benign tumors) or by advanced laparoscopy (16 malignant tumors). Ten (6.7\%) tumors were considered as "low-risk" but scheduled for primary laparotomy because of size $\geq 10 \mathrm{~cm}$ (9 benign and 1 malignant). Sensitivity, specificity, PPV and NPV for this scoring system were $98.6 \%, 94.9 \%, 94.7 \%$ and $98.7 \%$, respectively. The scoring system were more sensitive than patient's complaints $(98.6 \%$ vs $79.5 \%, \mathrm{p}<0.0001)$ and more specific than physical examination ( $94.9 \%$ vs $85.9 \%$, p $<0.0001$ )

Conclusions: Ultrasound based triage of symptomatic women diagnosed, as having an adnexal mass is effective for selecting surgical approach.
\end{abstract}

Keywords: Adnexal mass, ovarian cancer, complaints, ultrasound.

\section{INTRODUCTION}

Laparoscopy has become the gold standard in the surgical management of benign adnexal masses because this technique has a lower morbidity and shorter hospital stay as compared with laparotomy. ${ }^{1}$

On the other hand, accurate surgical staging and cytoreductive surgery have been shown to be among the most important prognostic factors in ovarian cancer. ${ }^{2}$ Furthermore, it has been demonstrated that better outcomes will be achieved for ovarian cancer patients when a gynecologic oncologist performs this surgery. ${ }^{3}$ For these reasons, women presenting with suspicious adnexal masses should be referred for primary laparotomy to specialized centers for gynecologic oncology with experienced surgeons and adequate resources.

However, many patients with ovarian cancer are still operated upon in local nonspecialized hospitals. ${ }^{4}$ In many cases this occurs because of an inaccurate preoperative diagnosis. Therefore, an accurate preoperative diagnosis is essential in order to establish the optimal management in these patients.

Ultrasound has been shown to be the best diagnostic tool for differentiating benign from malignant adnexal masses, with a sensitivity around $90 \%$ and a false-positive rate around $25 \%{ }^{5}$

Due to this fact, ultrasound is considered as the first step imaging technique to be used when assessing an adnexal mass. ${ }^{6}$

In 2003 we published our ultrasound scoring system, based on a morphologic and Doppler assessment, for distinguishing between benign and malignant adnexal masses ${ }^{7}$ which has been shown to be useful in asymptomatic patients. ${ }^{8}$

However, the performance of ultrasound based triaging could be different in symptomatic women because of many patient would complaint symptoms or would have a physical exam highly suspicious of ovarian cancer that could make ultrasound findings less relevant. As a matter of fact, recent research has pointed out that paying attention to patients' complaint could lead to an earlier diagnosis of ovarian cancer. ${ }^{9,10}$

The aim of the present study was to evaluate prospectively this scoring system as a method for triaging symptomatic women 
presenting with an adnexal mass for surgical treatment, laparoscopy or laparotomy.

\section{PATIENTS AND METHODS}

From June 2003 to December 2007, 151 symptomatic women diagnosed as having an adnexal mass and undergoing elective surgical treatment at our institution were included in this prospective study. Patients' mean age was 46 years, ranging from 15 to 82 years old. Ninety (60\%) women were premenopausal and sixty-one (40\%) were postmenopausal.

Our Institutional Review Board approved the study and all women gave written informed consent. All patients were evaluated within one week before surgery.

Diagnostic work-up included a complete medical history, physical examination and ultrasound examination.

Medical history focused especially in patients' complaints suggestive of ovarian cancer such as abdominal swelling, bloating and abdominal discomfort. Other symptoms recorded were abdominal and/or pelvic pain and uterine bleeding.

Staff specialists in Obstetrics and Gynecology, three of them specialized in gynecologic oncology (JLA, MJ, GLG), performed abdominal and vaginal physical examination in all cases previously to ultrasound examination. Findings were stated as:

- "Inconclusive", when no reliable information could be obtained.

- "Nonsuspicious", in the presence of a less than $8 \mathrm{~cm}$ maximum diameter adnexal mass, mobile at examination, of cystic or solid consistency but regular contours and no evidence of ascites

- "Suspicious", in the presence of at least one of the following: fixed and /or irregular adnexal mass regardless the size, a size $>8 \mathrm{~cm}$, evidence of ascites.

All patients were then evaluated by transvaginal powerDoppler ultrasonography using a Voluson 730 (GE, Milwaukee, IL, USA). No patient was excluded from the study. Scanning methodology was as follows:

Once the endovaginal probe was gently inserted into the vagina, the uterus and adnexal regions were scanned. Special attention was paid to adnexal masses. First tumor size was calculated according to maximum diameter. Then, morphological evaluation was performed attending the following parameters. ${ }^{11}$

- Bilaterality

- Wall thickness (thin $<3 \mathrm{~mm}$, thick $\geq 3 \mathrm{~mm}$ )

- $\quad$ Septations (not present, thin $<3 \mathrm{~mm}$, thick $\geq 3 \mathrm{~mm}$ )

- Papillary projections (not present, thin $<3$ mm length, thick $\geq 3 \mathrm{~mm}$ length)

- $\quad$ Solid areas (not present, presence of any solid area $\geq 1 \times$ $1 \mathrm{~cm}$ in internal wall surface or septum)

- Echogenicity

- Cystic: Anechoic, homogeneous content, heterogeneous content.

- Mostly solid: More than $80 \%$ of the tumor was solid.
After morphological evaluation was performed, power Doppler gate was activated to identify vascular color signals within the tumor (Doppler settings: frequency: $5 \mathrm{MHz}$, Power Doppler gain: 0.8, dynamic range: 20-40 dB, edge: 1, persistence: 2, color map: 5, gate: 2, filter: L1, PRF: $0.6 \mathrm{kHz}$ ). If blood flow was detected it was stated as "peripheral" (color signals in tumor wall or periphery of a solid tumor) or "central" (blood flow detected in septa, papillary projections, solid areas or central part of a solid tumor). In tumors with both peripheral and central blood flow only central blood flow was used for analysis.

Once a vessel was identified by color Doppler pulsed Doppler gate was activated to obtain a flow velocity waveform $($ FVW $)$. Resistance index $(\mathrm{RI}=[\mathrm{S}-\mathrm{D}] / \mathrm{S})$ and peak systolic velocity (PSV, cm/sec) were automatically calculated from at least three consecutive FVWs. In those tumors with more than one vessel the lowest RI and highest PSV found were used for analysis. Tumors were classified in four "velocimetric categories". ${ }^{12}$

- Low velocity/Low resistance (PSV $<10 \mathrm{~cm} / \mathrm{sec} / \mathrm{RI} \leq 0.45)$

- Low velocity/High resistance (PSV $<10 \mathrm{~cm} / \mathrm{sec} / \mathrm{RI}>0.45)$

- High velocity/High resistance (PSV $\geq 10 \mathrm{~cm} / \mathrm{sec} / \mathrm{RI}>0.45)$

- High velocity/Low resistance (PSV $\geq 10 \mathrm{~cm} / \mathrm{sec} / \mathrm{RI} \leq 0.45$ ).

All premenopausal women were evaluated in the follicular phase of the menstrual cycle.

According to findings our scoring system ${ }^{7}$ was applied (Table 1). A score $\geq 6$ was considered as suspicious for malignancy.

Table 1: Ultrasound-based scoring system

\begin{tabular}{ccccc}
\hline & $\begin{array}{c}\text { Thick papillary } \\
\text { projections }\end{array}$ & Solid areas & $\begin{array}{c}\text { Blood flow } \\
\text { location }\end{array}$ & $\begin{array}{c}\text { Doppler } \\
\text { velocimetry }\end{array}$ \\
\hline 0 & No & No & $\begin{array}{c}\text { No flow or } \\
\text { peripheral }\end{array}$ & No flow/Other \\
2 & Yes & - & - & $\begin{array}{c}\text { High velocity/Low } \\
\text { resistance }\end{array}$ \\
4 & - & Yes & Central & - \\
\hline
\end{tabular}

Patients were managed according to the following protocol (Fig. 1). Patients with adnexal masses with a score $<6$ and less than $10 \mathrm{~cm}$ in size were considered as "low-risk" for malignancy and were scheduled for laparoscopic surgery. Patients with adnexal masses with a score $<6$, but $\geq 10 \mathrm{~cm}$ sized and / or suspicion of pelvic adhesions were considered as "low-risk" for cancer but scheduled to laparotomy. Patients with adnexal masses with a score $\geq 6$ were considered as "high-risk" for cancer and were scheduled for laparotomy. In this group an abdominal ultrasound or CT scan was performed in order to determine the presence of spread disease. In this group, some selected cases (suspicious of metastatic tumor to the ovary, suspicion of noncytoreducible advanced stage ovarian cancer, suspicion of small-sized early ovarian cancer) were ultimately 


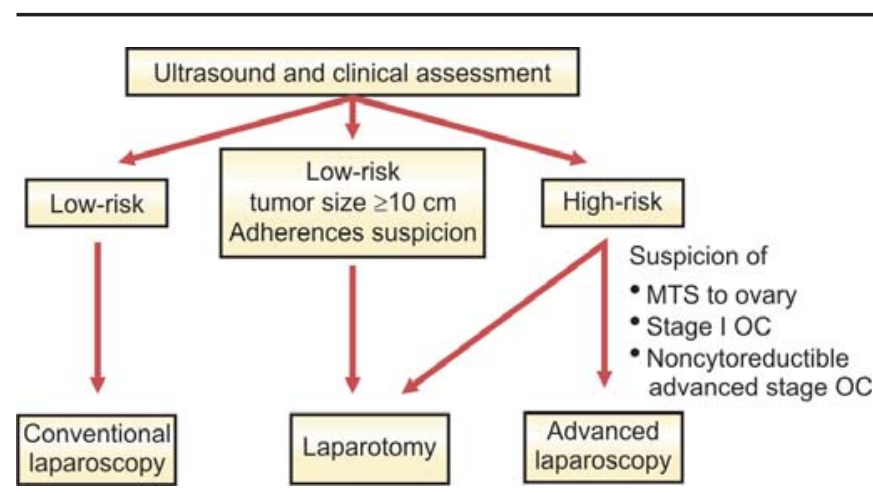

Fig. 1: Ultrasound-based triage for selecting surgical approach

scheduled for advanced oncologic laparoscopic surgery according to gynecologic oncologist surgeons' decision.

In all cases tumors were surgically removed by trained surgeons with more than 7 years experience in laparoscopic surgery (JLA, MJ, GLG, JAM, MGM) as well as more than 10 years experience in gynecologic oncology surgery (JLA, MJ, GLG) and definitive histological diagnosis obtained. Tumors were classified according to the World Health Organization. ${ }^{13}$ Ovarian malignancies were staged according to the FIGO. ${ }^{14}$ Low malignant potential tumors were considered as malignant.

Qualitative variables were described as percentages and compared by $\chi^{2}$ test. Continuous variables were presented as median and range and compared by U Mann-Whitney test.

Sensitivity, specificity, positive predictive value (PPV), negative predictive value (NPV) and accuracy were calculated for symptoms, physical examination and scoring system. McNemar test was used for comparing sensitivity and specificity.

A p value $<0.05$ was considered as statistically significant. SPSS 15.0 for Windows was used for statistical analysis.

\section{RESULTS}

Thirty women had bilateral tumors, however only one tumor that most suspicious on ultrasound - per case was used for analysis.

Overall, seventy-eight (52\%) tumors were benign and seventy-three (48\%) were malignant (Table 2).

Eighty-two women presented with pelvic pain, 8 had uterine bleeding and 61 referred symptoms suggestive for ovarian malignancy, such as abdominal swelling, bloating and abdominal discomfort (Table 3).

Physical examination was suspicious in 82 cases (54\%). Ultrasound score was $\geq 6$ in 72 out of 82 (88\%) cases (Table 4).

After ultrasound examination, sixty-five (43\%) masses were considered as "low-risk" and were scheduled for laparoscopy. In seven (16.3\%) of these women laparoscopy was converted to laparotomy because of pelvic adhesions all of them associated to severe pelvic endometriosis. All tumors in this group proved to be benign.
Table 2: Tumors' histology

\begin{tabular}{lrr}
\hline & $N$ & $\%$ \\
\hline Ovarian cancer stage I & 6 & 4.0 \\
Ovarian cancer stage II-IV & 56 & 37.1 \\
LMP tumor & 1 & 0.7 \\
Metastatic tumor & 10 & 6.6 \\
Endometrioma & 36 & 23.8 \\
Dermoid cyst & 10 & 6.6 \\
Serous cystadenoma & 4 & 2.6 \\
Mucinous cystadenoma & 6 & 4.0 \\
Hemorrhagic cyst & 8 & 5.3 \\
Hydrosalpinx & 3 & 2.0 \\
Cystadenofibroma & 2 & 2.0 \\
Tubo-ovarian abscess & 5 & 3.4 \\
Other benign tumors & 4 & 2.6 \\
\hline Total & 151 & 100 \\
\hline
\end{tabular}

Table 3: Symptoms according to histology

\begin{tabular}{lcc}
\hline & Benign & Histology \\
& Malignant \\
\hline Nonsuggestive OC & 75 & 15 \\
Suggestive OC & 3 & 58 \\
\hline
\end{tabular}

OC: Ovarian cancer

Table 4: Pelvic exam according to histology

\begin{tabular}{lcc}
\hline & \multicolumn{2}{c}{ Histology } \\
& Benign & Malignant \\
\hline Nonsuggestive OC & 67 & 2 \\
Suggestive OC & 11 & 71 \\
\hline
\end{tabular}

OC: Ovarian cancer

Seventy-six (50\%) masses were considered as "high-risk" tumors. In this group, sixty patients were scheduled for primary laparotomy (56 tumors were malignant and four were benign). Sixteen (21\%) women were scheduled for advanced oncologic laparoscopy due to suspicion of metastatic tumor to the ovary $(\mathrm{n}=5,31 \%)$, noncytoreducible advanced ovarian cancer $(\mathrm{n}=9$, $56 \%)$ and early stage ovarian cancer $(n=2,13 \%)$. All these tumors were malignant.

Ten (7\%) tumors were considered as "low-risk" but scheduled for primary laparotomy due to large size. In this group, nine (90\%) tumors were benign and one (10\%) was malignant. These results are summarized in Figure 2.

The correlation between ultrasound-based risk group and final histology is shown in Table 5.

The sensitivity, specificity, positive likelihood ratio and negative likelihood ratio of our scoring system, patients' complaints and physical examination are shown in Table 6. The scoring system was more sensitive than patient's complaints (98.6\% vs $79.5 \%, \mathrm{p}<0.0001$ ) and more specific than physical examination ( $94.9 \%$ vs $85.9 \%$, $\mathrm{p}<0.0001)$. 


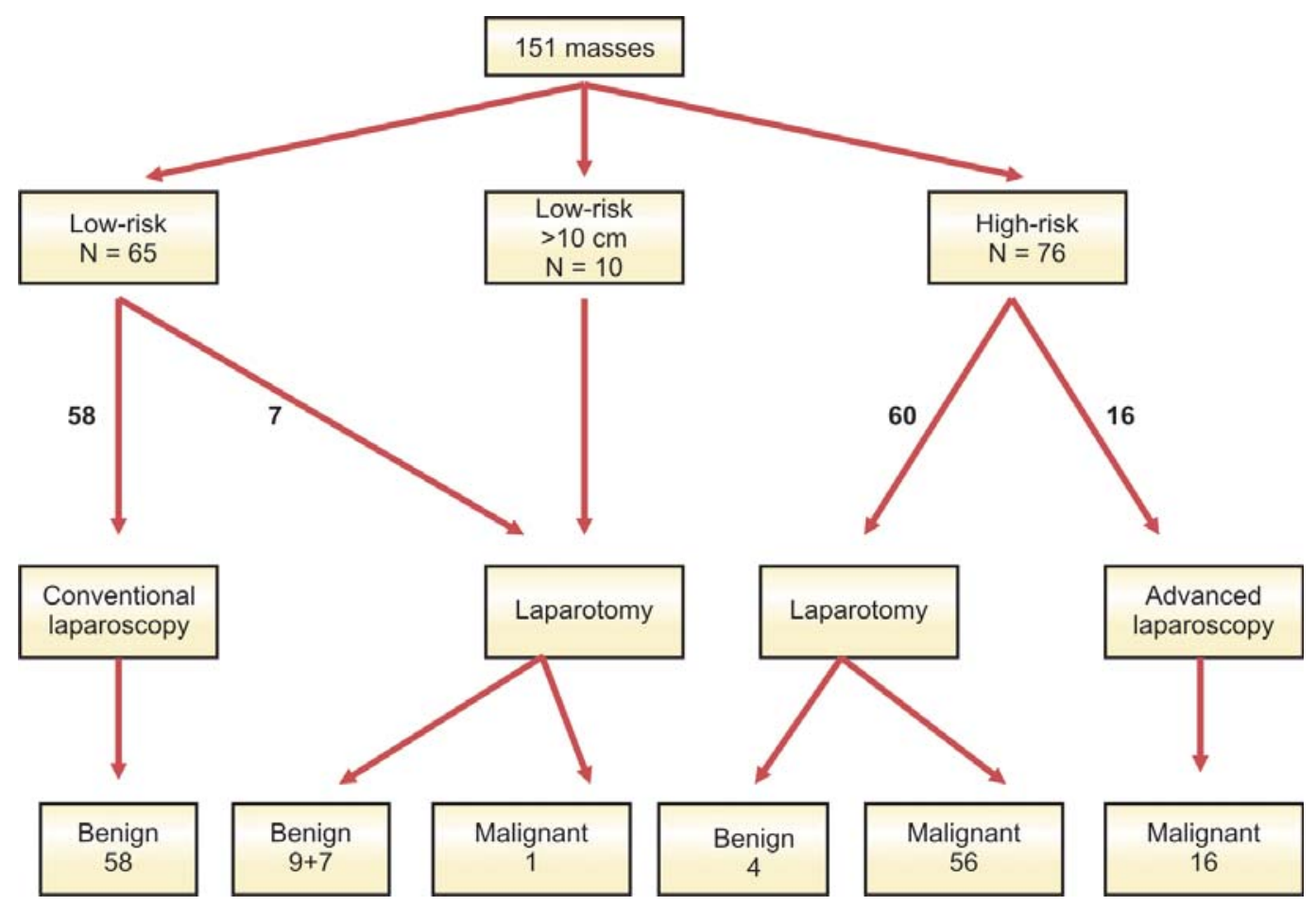

Fig. 2: Patients management according to our ultrasound and clinical triage

Table 5: Ultrasound score according to histology

\begin{tabular}{lcc}
\hline & \multicolumn{3}{c}{ Histology } \\
& Benign & Malignant \\
\hline Low-risk & 74 & 1 \\
High-risk & 4 & 72 \\
\hline
\end{tabular}

OC: Ovarian cancer

\section{DISCUSSION}

A woman with an adnexal mass still represents a challenge for clinicians because of the uncertainty about whether the patient should be referred for specialty surgical care. An accurate preoperative evaluation of adnexal masses is essential in order to establish the optimal management and appropriate referral for these patients.

Table 6: Diagnostic performance ultrasound, symptom evaluation and pelvic examination

\begin{tabular}{lccc}
\hline & Ultrasound & Symptom evaluation & Pelvic examination \\
\hline Sensitivity & $98.6 \%$ & $79.5 \%$ & $97.3 \%$ \\
& $95 \%$ CI: 93 to $100 \%$ & $95 \%$ CI: 69 to $87 \%$ & $95 \%$ CI: 90 to $99 \%$ \\
Specificity & $94.9 \%$ & $96.2 \%$ & $85.9 \%$ \\
& $95 \%$ CI: 87.5 to $89 \%$ & $95 \%$ CI: 89 to $99 \%$ & $95 \%$ CI: 76 to $92 \%$ \\
LR+ & 19.2 & 20.6 & 6.9 \\
& $95 \%$ CI: 7.4 to 49.9 & $95 \%$ CI: 6.8 to 63.0 & $95 \%$ CI: 3.9 to 11.9 \\
LR- & 0.014 & 0.21 & 0.03 \\
& $95 \%$ CI: 0.002 to 0.10 & $95 \%$ CI: 0.14 to 0.34 & $95 \%$ CI: 0.01 to 0.12 \\
\hline
\end{tabular}

LR+: Positive likelihood ratio

LR-: Negative likelihood ratio

95\%CI: 95\% confidence interval 
Ultrasound has been largely used in the differential diagnosis of adnexal masses and its diagnostic value is well-established. ${ }^{5}$

Most studies assessing the role of ultrasound for surgical or referral triaging of adnexal masses have been performed in series in which clinical complaints were not taking into account. ${ }^{15-19}$ It could be speculated that the performance of this diagnostic technique could be different in women complaining symptoms suggestive of ovarian cancer.

As a matter of fact, recent studies have shown that paying attention to patient's complaints may lead to an increased detection of ovarian cancer because of the identification of women at higher risk for this disease. ${ }^{9,10}$ Therefore, the risk for ovarian cancer is different in symptomatic patients as compared with asymptomatic women.

A similar situation could happen depending on the findings of pelvic examination. Physical examination has been shown to be of limited value for detecting adnexal masses, even under ideal circumstances. ${ }^{20}$ Furthermore, a recent meta-analysis highlighted the limitations of pelvic exam for predicting ovarian cancer in women diagnosed as having an adnexal mass. ${ }^{21}$ However, in some circumstances such as massive ascites the suspicion is very high.

We have shown that ultrasound is useful for surgical triaging in asymptomatic women. ${ }^{8}$ However, we wondered whether this technique could be also useful in a different clinical setting: symptomatic women.

The results of the present study reveal that ultrasound performs better than pelvic examination and symptoms evaluation for predicting ovarian cancer in symptomatic women with adnexal masses.

Ultrasound was more sensitive than symptom evaluation. This could be explained by the fact than some patients with ovarian cancer did not refer symptoms suggestive of ovarian cancer but other symptoms such as pelvic pain. "Pelvic pain" is actually a "broad" symptom that requires a thorough and specific evaluation. Some "kinds" of pelvic pain may be suggestive of ovarian cancer but others not. In fact, in our study most premenopausal (88\%) and many of postmenopausal (48\%) women that referred pain had benign lesions. On the other hand, pelvic exam was less specific than ultrasound. This could be explained by the fact that many benign tumors may exhibit large size or fixation at pelvic examination.

We had a false-negative case. This case was a stage IIIb mucinous ovarian cancer. Ultrasound examination showed a $6 \mathrm{~cm}$ solid tumor without blood flow. There were four falsepositive cases: One mucinous cystadenoma and three tuboovarian abscesses. In all four cases ultrasound examination showed a multilocular cystic mass with solid areas and blood flow within solid areas. Actually, in two cases of tubo-ovarian abscesses clinical presentation (sexually active premenopausal women complaining fever and pelvic pain) lead to a correct diagnosed. However, a strict application of the scoring system would provide a "suspicious" mass. It could be argued that these two cases might not be considered as false-positive cases when taken into account clinical presentation.

In conclusion, we have shown that ultrasound based triage of symptomatic women diagnosed as having an adnexal mass is effective for selecting surgical approach.

\section{REFERENCES}

1. Canis M, Rabischong B, Houlle C, Botchorishvili R, Jardon K, Safi A, Wattiez A, Mage G, Pouly JL, Bruhat MA. Laparoscopic management of adnexal masses: A gold standard? Curr Opin Obstet Gynecol 2002;14:423-28.

2. Levin L, Lund B, Heintz AP. Advanced ovarian cancer. An overview of multivariate analyses of prognostic variables with special reference to the role of cytoreductive surgery. Ann Oncol 1993;4:23-29.

3. Engelen MJ, Kos HE, Willemse PH, Aalders JG, de Vires EG, Shaapveld M, Otter R, van der Zee Ag. Surgery by consultant gynecologic oncologists improves survival in patients with ovarian carcinoma. Cancer 2006;106:589-98.

4. Carney ME, Lancaster JM, Ford C, Tsudikov A, Wiggins CL. A population-based study of patterns of care for ovarian cancer: Who is seen by a gynecologic oncologist and who is not? Gynecol Oncol 2002;84:36-42.

5. Kinkel K, Hricak H, Lu Y, Tsuda K, Filly RA. US characterization of ovarian masses: a metaanalysis. Radiology. 2000;217:803-11.

6. Mettler L. The cystic adnexal mass: patient selection, surgical techniques and long-term follow-up. Curr Opin Obstet Gynecol 2001;13:389-97.

7. Alcazar JL, Merce LT, Laparte C, Jurado M, López-García G. A new scoring system to differentiate benign from malignant adnexal masses. Am J Obstet Gynecol 2003;188:685-92.

8. Alcázar JL, Royo P, Jurado M, Mínguez JA, García-Manero M, Laparte C, Galván R, López-García G. Triage for surgical Management of ovarian tumors in asymptomatic women: assessment of an ultrasound-based scoring system. Ultrasound Obstet Gynecol 2008;32:220-25.

9. Goff BA, Mandel LS, Drescher CW, Urban N, Gough S, Schurman KM, Patras J, Mahony BS, Andersen MR. Development of an ovarian cancer symptom index: Possibilities for earlier detection. Cancer 2007;109:221-27.

10. Andersen MR, Goff BA, Lowe KA, Scholler N, Bergan L, Dresher CW, Paley P, Urban N. Combining a symptoms index with CA 125 to improve detection of ovarian cancer. Cancer 2008;113:484-89.

11. Timmerman D, Valentin L, Bourne TH, Collins WP, Verrelst $\mathrm{H}$, Vergote I. Terms, definitions and measurements to describe the sonographic features of adnexal tumors: A consensus opinion from the International Ovarian Tumor Analysis (IOTA) Group. Ultrasound Obstet Gynecol 2000;16:500-05.

12. Alcázar JL, Ruiz-Pérez ML, Errasti T. Transvaginal color Doppler sonography in adnexal massess: Which parameter performs best? Ultrasound Obstet Gynecol 1996; 8:114-19. 
13. Serov SF, Scully RE, Sobin LH. International histological classification of tumors. No. 9, Histological typing of ovarian tumors, World Health Organization, Geneva 1973.

14. Sheperd JH. Revised FIGO staging for gynecological cancer. Br J Obstet Gynecol 1989;96:889-92.

15. Berlanda N, Ferrari MM, Mezzopane R, Boero V, Grijuela B, Ferrazzi E, Pardi G. Impact of a multiparameter, ultrasoundbased triage on surgical management of adnexal masses. Ultrasound Obstet Gynecol 2002;20:181-85.

16. Guerriero S, Ajossa S, Garau N, Piras B, Paoletti AM, Melis GB. Ultrasonography and color Doppler-based triage for adnexal masses to provide the most appropriate surgical approach. Am J Obstet Gynecol 2005;192:401-16.

17. Bailey J, Tailor A, Naik R, Lopes A, Godfrey K, Hatem HM, Monaghan J. Risk of malignancy index for referral of ovarian cancer cases to a tertiary center: does it identify the correct cases? Int J Gynecol Cancer 2006;16:30-34.

18. Yazbek J, Helmy S, Ben-Nagi J, Holland T, Sawyer E, Jurkovic D. Value of preoperative ultrasound examination in the selection of women with adnexal masses for laparoscopic surgery. Ultrasound Obstet Gynecol 2007;30:883-88.

19. Chia YN, Marsden DE, Robertson G, Hacker NF. Triage of ovarian masses. Aust N Z J Obstet Gynaecol 2008;48:322-28.

20. Padilla LA, Radosevich DM, Milad MP. Limitations of the pelvic examination for evaluation of the female pelvic organs. Int $\mathrm{J}$ Gynaecol Obstet 2005;88:84-88.

21. Myers ER, Bastian LA, Havrilesky LJ, Kulasingam SL, Terplan MS, Cline KE, Gray RN, McCrory DC. Management of adnexal mass. Evid Rep Technol Assess (Full Rep) 2006;130:1-145. 